\title{
A Research on Security, Privacy Issues and Privacy Preserving Techniques - Big Data
}

\author{
G.Rama Devi, Y.Rajesh Babu
}

\begin{abstract}
Information, which is not a fear till 2000, was the precept criteria for man or woman regarding safety and protection a brief time later. these days enormous measure of information is being created for always it is a check to deal with it. it is being used by numerous groups to take large options from the beyond facts to interrupt down thefuture. This tremendous diploma of facts is referred to as as huge statistics. in spite of the fact that there are numerous common calculations in presence, for giving safety and safety in large records it's miles attending to be tough because of its traits (quantity, range and Veracity).

This paper demonstrates the investigation of common techniques handy, troubles to protection and safety of big records and protection saving processes like anonymization, randomization, differential safety, word and assent and so on.
\end{abstract}

Keywords:- Big data, Data privacy and Security challenges, Privacy Preserving Techniques.

\section{INTRODUCTION}

Statistics is growing exponentially with time. In a word, such facts is excessively massive and complex that none of the conventional data the board devices can keep it or method it efficaciously. This information might be tested computationally to find out examples, styles, and affiliations, specifically figuring out with human behavior and connections. trends of massive facts huge facts have three good sized attributes (Fig. 1):

- speed: How quick statistics is being produced

- variety: belongings from which information is being created

- extent: duration of the facts produced

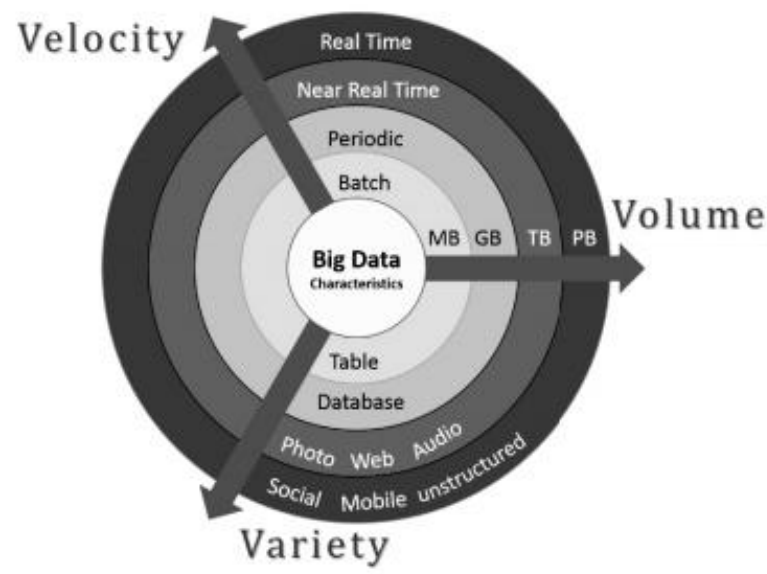

Fig.1. Characteristics of Big Data

Following are a part of the instances of large information-

Revised Manuscript Received on April 12, 2019.

G.Rama Devi, Asst.Prof, Dept. of CSE, VasireddyVenkatadri Inst. of Tech., Guntur, AP,India. (ramarajesh95@gmail.com)

Y.Rajesh Babu, Asst.Prof, Head, Dept. of CSE, Priyadarshini Inst. of Tech. and Sc. (W), Tenali, AP, India. (raj.urs18@gmail.com)
- The big apple inventory trade (creates spherical one TB of latest facts every day).

- facebook (the scale demonstrates that 500+ TB of recent facts is introduced to the databases of 8db290b6e1544acaffefb5f58daa9d83 networking website fb, continuously). This information incorporates pictures, recordings, immediate message and so on.

- The single Jet motor: hundreds of flights run for every day, and age of information reaches as much as numerous Petabytes (it produces $10+$ TB of statistics in half-hour).

\section{Training of large information}

Advanced facts has visible exponential development in the previous decade or someplace within the location, which is called prepared and unstructured statistics. organized information is profoundly composed and carries tables that represent their significance. E.g., Excel spreadsheets and social databases. Unstructured information is the whole thing else. as an instance right now messages, content material statistics, sound and video transcripts PowerPoint, Slide share introductions, Audio documents of track cellphone messages, Video information, images, outon lines, pictures, and so on.

Unstructured facts represents over $90 \%$ of all statistics as appeared in determine 2 .

"Among the start of human development and 2003, we have produced simply five Exabyte of records however now it's far the diploma of data being made for each two days. by 2020 , it's miles predicted that round 53 zettabytes (for example 53 trillion gigabytes) of statistics may be produced this is an expansion of multiple times." - Hal Varian, chief Economist at Google.

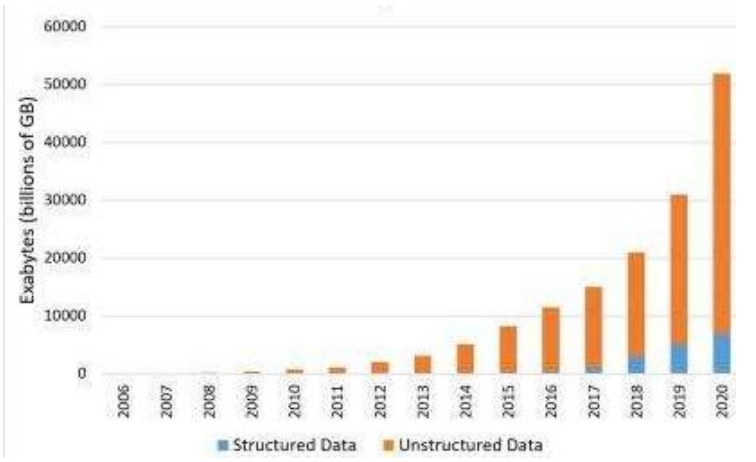

Fig.2. the growth of structured vs.unstructured data over the past decade 
IBM found that now humans are creating 2.5 quintillion bytes of data daily; that's the equivalent of about half a billion HD movie downloads as described in Table 1.

Table1. Examples of prefixes used to measure digital data with a binary system

\begin{tabular}{|l|l|l|l|l|}
\hline $\begin{array}{l}\text { Multiplying } \\
\text { factor }\end{array}$ & SI Prefix & Decimal & $\begin{array}{l}\text { Binary } \\
\text { Prefixes }\end{array}$ & Name \\
\hline $\begin{array}{l}1,208,925,81 \\
9,614,629,17 \\
4,706,176\end{array}$ & Yottabytes & $10^{24}$ & $2^{80}$ & $\begin{array}{l}1 \\
\text { septillion }\end{array}$ \\
\hline $\begin{array}{l}1,180,591,62 \\
0,717,411,30 \\
3,424\end{array}$ & Zettabytes & $10^{21}$ & $2^{70}$ & $\begin{array}{l}1 \\
\text { sextillion }\end{array}$ \\
\hline $\begin{array}{l}1,152,921,50 \\
4,606,846,97 \\
6\end{array}$ & Exabytes & $10^{18}$ & $2^{60}$ & $\begin{array}{l}1 \\
\text { quintillio } \\
\mathrm{n}\end{array}$ \\
\hline $\begin{array}{l}1,125,833,90 \\
6,842,624\end{array}$ & Petabytes & $10^{15}$ & $2^{50}$ & $\begin{array}{l}1 \\
\text { quadrilli } \\
\text { on }\end{array}$ \\
\hline $\begin{array}{l}1,099,511,62 \\
7,776\end{array}$ & Terabytes & $10^{12}$ & $2^{40}$ & 1 trillion \\
\hline $\begin{array}{l}1,073,741,82 \\
4\end{array}$ & Gigabytes & $10^{9}$ & $2^{30}$ & 1 billion \\
\hline $1,048,576$ & Megabytes & $10^{6}$ & $2^{20}$ & 1 million \\
\hline 1,024 & Kilobytes & $10^{3}$ & $2^{10}$ & $\begin{array}{l}1 \\
\text { thousand }\end{array}$ \\
\hline
\end{tabular}

\section{What is Analytics?}

Research is a multi-dimensional and along with place. It utilizes prescient displaying, era, measurements and AI systems to discover vital examples and facts from recorded information [19].

What's large statistics Analytics?

Large information examination is a thoughts boggling method of analyzing the extraordinary collection of informational indexes called big facts to break down difficult to understand facts like shrouded designs, hard to understand relationships, advertise styles and purchaser inclinations which assist associations to pick industrial corporation selections [2]. With using big records examination, you probable can determine knowledgeable selections with out indiscriminately depending on suppositions. what is greater, it solutions the accompanying forms of inquiries:

- What truly took place?

- How or for what reason did it get up?

- what's going on now?

- What is probably going to arise straightaway?

Massive records Analytics is utilized in one in all a kind businesses like Banking, government, fitness Care, Retail, manufacturing, education and so forth.

massive information rather than large facts Analytics more regularly than not, records studies is extra engaged than huge facts. instead of social event fantastic heaps of unstructured information, information examiners set a specific goal at the pinnacle of the priority listing and kind in like way just the pertinent facts to look for strategies to pick out up help. however, exquisite records is an accumulation of a giant volume of statistics which calls for a ton of sifting through to get beneficial bits of information from it.

Every other key evaluation is that big facts utilizes complex cutting-edge gear[15] like parallel processing and other computerization devices to cope with the "massive statistics". data examination make use of prescient and actual showing with normally fundamental units.

\section{Characterization of huge information}

Big statistics is being arranged into precise schooling which might be to be considered in lifestyles cycle of massive facts as appeared in determine 3 .

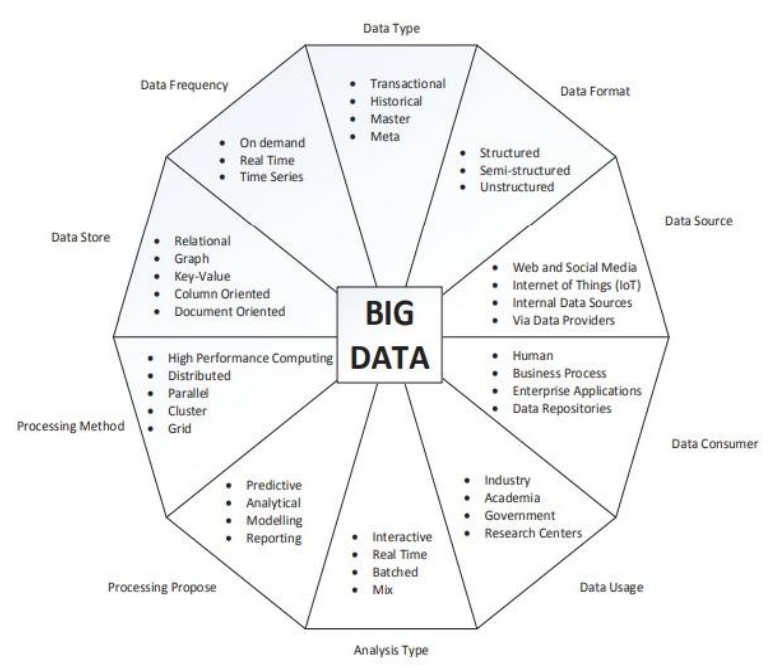

Fig.3. Big Data Classification

Traditional techniques like cryptography may be used but they do not display to be green due to the complex nature of information. information anonymization or De-identification is a manner of hiding private statistics. it's far the machine of converting touchy records when publishing the records. There are three information anonymization techniques that are used in keeping big information privacy. they will be okay-Anonymity, L-variety, and T-Closeness.

extraordinary privacy preserving strategies to be had are phrase and Consent, Differential privateness, Randomization [3].

2. privacy AND protection demanding situations $\mathrm{OF}$ huge records Programs had been designed to offer safety for storing information (now not for huge volumes of records). however these packages were proved inefficient to preserve dynamic statistics. therefore, only a safety check can not come across protection issues for continuous streaming of data. And as a result, it is wanted a complete-time privateness check while statistics streaming and large facts evaluation.

There are mainly ten worrying conditions [4] [5 - 7] inside the subject of big information security and privacy as mentioned underneath

1) Protecting Transaction Logs and statistics

Garage management is a key part of the large facts security. The CSA recommends using signed message 
digests that gives a virtual identifier for each virtual record or document and the usage of comfortable untrusted data repository approach (SUNDR) to hit upon unauthorized report modifications by means of using malicious server marketers. There are different strategies like lazy revocation and key rotation, broadcast and policy primarily based encryption schemes, and digital rights manipulate (DRM). however, there can be no alternative for virtually constructing our very personal comfy cloud storage on top of cutting-edge infrastructure.

2) Validation and Filtration of stop-point Inputs

Stop-factor devices play a key characteristic in maintaining big facts. garage, system and special essential responsibilities place unit performed with the assist of the enter file, this is provided via way of quit-factors. therefore, an employer want to ensure to use an right and valid ceasething device.

three) Securing dispensed Framework Calculations and other methods

Computational protection and other digital property in a dispensed framework just like the Map-reduce function of Hadoop, lack safety. the 2 most important preventions for it are securing the mappers and protecting the data from an unauthorized mapper.

four) Securing and protecting records in real Time

Due to massive quantities of data era, maximum businesses aren't able to hold ordinary tests. however, it's most useful to carry out protection assessments and announcement in actual time.

5) protecting get entry to manipulate method communication and Encryption

A secured data storage device is an sensible step for protective the information. but, due to most regularly used information garage gadgets region unit is inclined, it's far vital to encrypt the access manage methods as nicely.

6) information Provenance

To categorise facts, it's miles crucial to be aware of its foundation [13]. on the way to decide the facts beginning place as it should be, authentication, validation and get access to manipulate can be obtained and implement dynamic and scalable granular get admission to controls and put in force encryption strategies

7) Granular Auditing

Analyzing awesome sorts of logs will be great and this records might be beneficial in figuring out any moderately cyber-assault or malicious hobby. consequently, ordinary auditing can be beneficial.

8) Granular get admission to control

Access manipulate is ready center topics in line with the CSA: proscribing consumer access and granting the consumer access. It is right to construct and positioned into impact a policy that chooses the right one in any given state of affairs. For putting in granular get admission to controls, the CSA has a gaggle of brief-hit guidelines:

o Normalize mutable factors and De-normalize immutable factors

o tune secrecy requirements and make certain right implementation

o hold access labels

o music admin records

o Use unmarried signal-on (SSO) and o Use a labelling scheme to hold right data federation.

9) privacy safety for Non-Rational facts shops

Records stores which includes NoSQL have many protection vulnerabilities, which motive privateness threats. An awesome safety flaw it's unable to write facts in the course of the tagging or work of understanding or whereas meting out it into totally specific organizations as soon as it is streamed or amassed.

three. privacy THREATS IN data ANALYTICS

Privacy is in arms of the individuals and to ensure about what facts to be shared, and additionally need to have manage on it. If the statistics is in public domain names then it is truly a risk [8] to person privateness as the statistics is being held via the data holder. information holder can be everybody like social networking application, web sites, cellular apps, e-trade web website, banks, hospitals and so forth. So, it's far the duty of the records holder to make certain privacy for every body's information. aside from this, customers knowingly or unknowingly make contributions themselves to data leakage. for example, most of the cellular apps, are searching out get right of access to to our contacts, files, digicam and so on. and without reading the privateness declaration we agree for all phrases and conditions, thereby data leakage occurs.

some of the critical element privateness threats which could attack every mobile phone

- Surveillance

Many companies like retail, e-exchange, and so on. will constantly screen their client's behavior. Ex: E-trade websites studies clients shopping for behavior and suggests the numerous offers, comparable items, exciting objects and charge-brought services that's a vital privateness threat.

- Disclosure

If we do not forget the statistics of a patient from a hospital which incorporates his/her call, gender, ailment, signs and symptoms and so forth. the facts holder will speak with the zero. 33 celebration for analysis from which private statistics isn't always disclosed. however this $1 /$ threecelebration facts analyst can add this records to be had to external statistics assets from which everybody can get the man or woman-precise statistics. this is how the non-public statistics may be disclosed [9] it's taken into consideration to as a intense privateness danger.

- Discrimination and private embracement and abuse This specially occurs while some private data of someone is disclosed.

lack of expertise is additionally a giant motive for privateness attacks. previous research indicates fine $17 \%$ of telephone users are aware about privateness threats.

\section{PRIVACY PRESERVING METHODS \& RESULTS}

Severa privateness saving tactics have been grown, yet most people of them depend upon the anonymization of information. here is a rundown of protection safeguarding methods [1] given below.

Blue Eyes Intelligence Engineering

\& Sciences Publication 
1) De-distinguishing proof or Anonymization techniques

o ok-secrecy

o L-diverse range

o T-closeness

2) Differential privateness

three) be aware and Consent

4) Randomization

5) records conveyance

6) Cryptographic techniques

7) decreasing

eight) MDBSA(Multidimensional Sensitivity primarily based Anonymization)

\subsection{De-identity or Anonymization techniques}

It's far the method used to maintain an character's man or woman from being associated with facts. regular structures for de-identifying[13][14] datasets comprise erasing or overlaying person identifiers, as an example, name and government managed savings variety, and smothering or summing up semi identifiers, for example, date of shipping and postal department.

The data in the database is probably categorised as pursues:

i. Explicit identifiers-the ones aides in spotting an character curiously e.g.: name, Aadhar, Roll No and so forth.

ii. Semi identifiers-these can be joined with special records for recognizing a person from the society's. e.g.: intercourse, age, city and so forth.

iii. Touchy identifiers-those are the trends with delicate incentive as for the records owner. model: illness, income, and so forth.

iv. Non-sensitive identifiers-the ones are the characteristics which do now not make any trouble however they're uncovered.

The anonymization consists of the accompanying methodologies:

I. speculation and Suppression - Generalization replaces the particular capabilities with more and more extensive ones that result in having many copy esteems [17]. In concealment, semi identifiers are supplanted with ordinary traits like zero, ${ }^{*}$ and so on.

ii. Anatomization and Permutation-This de-interfaces the connection amongst semi identifiers and sensitive trends.

iii. bother - It includes a variety of some clamor to the primary statistics in advance than distributing to the client.

Gatecrashers can surely separate the information from this de-recognizable proof

four.1.1 ok-Anonymity

A dataset is stated to be adequate-anonymized that for any given tuple having sure tendencies inside the dataset, there ought to be in any occasion okay-1 tremendous records that in shape those developments. this will be finished by using making use of concealment and hypothesis sports [1]. Following are the limitations, as delineated in Fig. 4. Constraints

1. Homogeneity-assault or characteristic linkage
2. basis facts. Or however report linkage

3. Computational complexity O (k log k)[10]

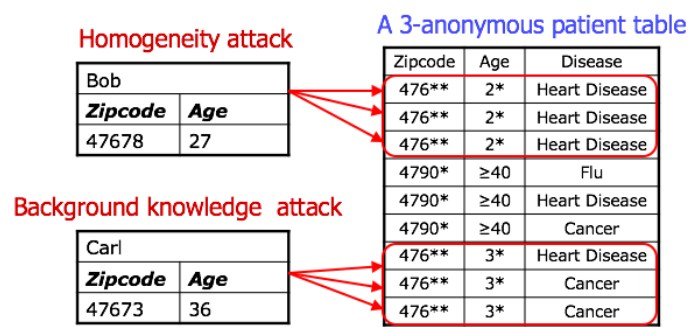

Fig.4. Homogeneity and Background Knowledge attack

\subsubsection{L-Diversity}

It's miles actualized to steer clear of from homogeneity assault and basis assault. in this technique, each sensitive characteristics are spoken to by means of nicely-spoken to values. To characterize "L very a great deal spoke to" values, every proportionality class need to have at any charge L unmistakable traits for the delicate field. that is referred to as distinct L-decent variety. Following are the limitations, as portrayed in Fig. 5.

Impediments

1. Skewness assault

2. Likeness assault

3. Computational multifaceted nature $\mathrm{O}((\mathrm{n} 2) / \mathrm{k})[11]$

\begin{tabular}{|c|c|c|c|c|c|}
\hline \multicolumn{2}{|c|}{ Similarity attack } & \multicolumn{4}{|c|}{ A 3-diverse patient table } \\
\hline & & Zipcode & Age & Salary & Disease \\
\hline \multicolumn{2}{|l|}{ Bob } & $476 * *$ & $2^{*}$ & $20 \mathrm{~K}$ & Gastric Ulcer \\
\hline Zip & Age & $\begin{array}{l}476^{* *} \\
476^{* * *}\end{array}$ & $\begin{array}{l}2^{*} \\
2^{*}\end{array}$ & $\begin{array}{l}30 \mathrm{~K} \\
40 \mathrm{~K}\end{array}$ & $\begin{array}{c}\text { Gastritis } \\
\text { Stomach Cancer }\end{array}$ \\
\hline \multirow[t]{5}{*}{47678} & 27 & $4790 *$ & $\geq 40$ & $50 \mathrm{~K}$ & Gastritis \\
\hline & & $\begin{array}{l}4790^{*} \\
4790^{*}\end{array}$ & $\begin{array}{l}\geq 40 \\
\geq 40\end{array}$ & $\begin{array}{l}100 \mathrm{~K} \\
70 \mathrm{~K}\end{array}$ & $\begin{array}{c}\text { Flu } \\
\text { Bronchitis }\end{array}$ \\
\hline & & $476 * *$ & $3^{*}$ & $60 \mathrm{~K}$ & Bronchitis \\
\hline & & $476 * *$ & 3* & $80 \mathrm{~K}$ & Pneumonia \\
\hline & & $476^{* *}$ & 3* & $90 \mathrm{~K}$ & Stomach Cancer \\
\hline
\end{tabular}

Fig.5. Similarity Attack

\subsubsection{T-Closeness}

This method is the development of both k-obscurity and 1decent range. It helps in ensuring the safety of datasets and furthermore reducing the granularity of an facts portrayal. within the event that the t-closeness tenet holds for a dataset, at that point we can say that it holds okay-namelessness and l-first rate range standards furthermore. The T-closeness degree is simplest the separation between the circulation of a delicate trait in this magnificence and the conveyance of the feature within the whole table which isn't in excess of an area t. Computational intricacy $2 \mathrm{O}(\mathrm{n}) \mathrm{O}(\mathrm{m})[12]$.

\subsection{Differential privateness}

Current strategies are there for giving security by way of concealing facts from gatecrashers yet these strategies cannot give the guarantee about the stowing away of records genuinely and therefore this differential safety method is accomplished. The essential point is to offer international, actual data which is overtly reachable whilst securing the ones clients safety whose records is contained inside the 
dataset. The origination of "in-noticeability", furthermore known as "differential safety", in the placing of linked mathematics databases. As it's miles a probabilistic concept, any differentially private device is fundamentally arbitrary. Differential security is a definition, now not a calculation. For a given methodology project $\mathrm{T}$ related a given value of there will be some differentially non-open calculations for engaging in $\mathrm{T}$ in a differentially non-open manner. some will have preferred precision over others. every time little, discovering amazingly right differentially non-open preferred is for $\mathrm{T}$ could be extreme, specifically like finding a numerically consistent principle for a particular method challenge will require exertion.

4.Three be aware and Consent

By way of this approach, purchaser information is shared absolutely subsequent to obtaining assent from the purchaser utilizing a observe. This strategy is typically utilized while a purchaser utilizes another utility or every other internet administration.

\section{Four Randomization}

Randomization is the way toward adding clamor to the facts that's typically accomplished by using the chance conveyance. Randomization is applied in opinion research, overviews and so on. It needn't hassle with records of different statistics within the data. It thoroughly can be linked at some stage in records collecting and pre-getting ready stage. there is no anonymization overhead in this technique. due to its time multifaceted nature and records application, it's far preposterous to anticipate to apply on large datasets

\subsection{Statistics Distribution}

Appropriation of the facts ought to be viable in two oneof-a-kind approaches, Horizontal and Vertical dissemination of information

Level dissemination: The conveyance is said to be even appropriation while the information is circulated crosswise over numerous locales with similar features at that point

Vertical circulate: The dispersion is stated to be vertical dissemination whilst person explicit information is appropriated crosswise over numerous locales beneath caretaker of various institutions

\subsection{Cryptographic strategies}

The goal of cryptography is to have secure correspondence (as an instance categorized and legitimate). This must be viable through concealing a few residences of facts and for that reason they're statistics self sufficient. Cryptography is normally wasteful with a variety of information. for instance, it's miles a trifling errand to get better the ithelement $\mathrm{Di}$ of a database $\mathrm{D}$. The cryptography primarily based technique for the most part ensures an extremely odd kingdom of statistics security. In Kantarcioglu and Clifton deal with, the issue of at ease mining of affiliation controls over on a stage aircraft parceled facts, using cryptographic systems to restrict the facts shared [20]. Their answer depends on the presumption that each collecting to begin with scrambles its personal thing sets utilising commutative encryption, at that factor the as of now encoded factor sets of each different collecting. later on, a starting collecting transmits its recurrence test, further to an arbitrary really worth, to its neighbor, which incorporates its recurrence tally and passes it directly to specific gatherings. At long remaining, a covered correlation happens between a definitive and starting gatherings to training session if a definitive outcome's larger than the brink and the irregular worth. Registering association policies even as now not uncovering singular exchanges is direct. we can procedure the global assist and truth of an association rule $\mathrm{AB} \Rightarrow \mathrm{C}$ knowing simply the close by backings of $\mathrm{AB}$ and $\mathrm{ABC}$, and the scale of each database

\subsection{Reducing}

Slicing is a method that breaks the relationship amongst segments (for instance uncorrelated characteristics), by means of saving the connection interior each segment [14]. The dimensionality of the facts is diminished and application is superior to hypothesis and Bucketization There are broadly applied information anonymization tactics especially Generalization and Bucketization. these systems are applied to security shielding small scale records distributing. It segments the dataset each vertically and on a level aircraft.

Vertical apportioning: based on the connections amongst features which are amassed into sections. each segment can have a subset of very associated houses.

Even parceling: bunches tuples into bins. additionally, inside every can, and in every segment, values are haphazardly permutated (or arranged) to interrupt dating among diverse sections.

four.eight MDBSA (Multidimensional Sensitivity based Anonymization)

Base up Generalization and top-down Specialization are the conventional strategies for Anonymization that's done on organized records [16] [18]. but, this could't be linked on enormous datasets productively because of adaptability and information misfortune. Multidimensional Sensitivity based Anonymization is an stepped forward shape of Anonymization. guide reduce shape has been applied to deal with big datasets in Apache. On delicate characteristics, backside-up Generalization is utilized and facts is vertically apportioned into various gatherings, it is able to defend from foundation mastering attack if the sack carries just multiple characteristics.

\section{CONCLUSION}

A complete outline of PPDM systems dependent on randomization, dispersion, and ok-anonymization are displayed. At present massive degree of data is being produced for always. it's miles being shared among different segments like well-being, government, casual organizations and so on. subsequently there may be a need to maintain up and make certain the facts with out letting realize the subtleties of delicate data. as a consequence, its quick development is confronting new difficulties to provide protection and safety to information. current strategies are not adequate to cope with the big statistics and finally there is a need to develop new strategies for taking care of the facts in destiny years. 


\section{REFERENCES}

1. Karim Abouelmehdi, Abderrahim Beni-Hessane, Hayat khaloufi "widespread social coverage records: safeguarding security and protection" journal of big information, December 2018, https://doi.org/10.1186/s40537-017-0110-7.

2. Duygu Sinanc Terzi, Ramazan Terzi, Seref Sagiroglu "A Survey on safety and privateness troubles in huge information" In Proc. of tenth Intl. Conf. for internet generation and Secured Transactions (ICITST), December 2015.

3. P. Slam Mohan Rao, S. Murali Krishna, A. P. Siva Kumar "security conservation methods in large records research: a top level view" journal of big facts, September 2018.

4. Cloud safety Alliance(CSA) Collaborative studies "extended top Ten huge facts safety and privacy demanding situations" April 2013.

5. Hervais Simo Fhom "A Technical report on huge data: possibilities and privateness challenges", Feb 2015.

6. A humans group White paper created by way of riding scientists crosswise over US, "problems andOpportunity with massive information", Feb. 2012.

7. Julio Moreno, Manuel A. Serrano, Eduardo FernandezMedina "primary troubles in large statistics protection", MPDI article.

8. M. Hettig, E Kiss, J.- F Kassel, S Weber, M Harbach, M Smith "Picturing hazard with the aid of example: Demonstrating Threats bobbing up From Android Apps" In Proc. of Symposium on Usable privateness and safety (SOUPS), Newcastle, united kingdom, July 2013.

9. Lambert Diane "Proportions of divulgence danger and mischief" journal of authority statistics.1993; nine(2):313.

10. Robert Bredereck, Andre Nichterlein, Rolf Niedermeier, Geevarghese Philip "The effect of homogeneity on the unpredictability of okay-namelessness". In Proc. of Intl. Symposium on basics of Computation theory, 2011. p. fifty three-64.

11. Machanavajjhala A, Gehrke J, Kifer D, VenkitasubramaniamM. "L-diverse variety: protection past kobscurity". In: Proc. 22nd established assembly records designing (ICDE); 2006DuncanGTetal.

12. Ninghui Li, Tiancheng Li, Suresh Venkatasubramanian "TCloseness: safety past okay-namelessness and L-respectable range", In Proc. of Intl. Conf. on information Eng., Istanbul, Turkey, 2007.

13. B. Matturdi, X. Zhou, S. Li, F. Lin, "giant data security and safety: An audit", huge statistics, Cloud and cellular Computing, China Communications vol.eleven, difficulty: 14, pp. $135-145,2014$

14. G. Rama Devi, A. Anuradha, "advanced slicing: An technique for keeping Correlations among sensitive Attributes" Intl. Diary of Computational Mathematical ideas,Vol.6, issue3,Nov-2014,pageno: 60841-60846.

15. G. Rama Devi, G. Pratyusha, P. Reshma "assessment on importance and equipment Used: BigData" Intl. Diary of advanced studies in laptop technology and software Engineering (IJARCSSE)Vol.6, issue-5May-2016, web page no513-516.

16. okay. Wang, P.S. Yu, S.Chakraborthy "Base up hypothesis: A facts mining solution for security coverage" In Proc. of the fourth IEEE international convention on information Mining (ICDM), Brighton, united kingdom November 2004.

17. Sweeney L "engaging in okay-secrecy security coverage making use of hypothesis and concealment "worldwide magazine on Uncertainty, Fuzziness and knowledge based totally structures, 2002, vol. 10 problem 5, p.no 571-588.

18. B.C.M. Fung, ok. Wang, P.S. Yu "top-down specialization for statistics and protection safeguarding" In Proc. of the21st international conference on statistics Engineering (ICDE'05), Japan, April 2005.

19. Xu k, Yue H, Guo Y, Fang Y. "protection safeguarding AI calculations for massive facts frameworks" In Proc. of thirty fifth IEEE global assembly on dispersed structures(ICDCS), July 2015, Columbus, Ohio, usa.

20. Chin-Chen Chang, Jieh-Shan Yeh, Yu-Chiang Li "safety preserving Minin 